\title{
Recuperação da vegetação de praia - Praia de Grumari, RJ
}

\author{
Janice Rezende Vieira PEIXOTO'; Rita de Cássia Martins MONTEZUMA'; \\ Marcos Aurélio PERRONI'; Allana Oliveira LIMA \\ 凹tartbr@yahoo.com.br
}

1. Universidade Federal Fluminense, Avenida General Milton Tavares de Souza, Rio de Janeiro, RJ.

\section{Histórico do Artigo:}

Recebido: 29 de março de 2016
Aceito: 23 de novembro de $2016 \quad$ Publicado: 22 de dezembro de 2016

\begin{abstract}
Resumo: Este trabalho visa avaliar duas técnicas de plantio da espécie Ipomoea pes-caprae (Convolvulaceae), propagação de estaquia e plantio por mudas, em uma área de Proteção Ambiental, Parque Natural Municipal de Grumari, RJ. Foram plantados 900 espécimes de Ipomea pes-capreae, transplantadas do horto Restinga, em agosto de 2015, distribuídas equitativamente entre os dois tipos de plantio: 450 mudas diretamente plantadas e 450 por propagação de estaquia. Nos meses de setembro (mês 1) e outubro (mês 2) de 2015, observou-se que a mortalidade foi de aproximadamente $20 \%$ para o plantio de mudas enraizadas e $70 \%$ para o plantio por estaquias. Já no mês de novembro (mês 3) foi observado um a mortalidade de $60 \%$ no plantio de mudas por enraizamento e $90 \%$ do plantio por estaquia. A partir de janeiro (mês 5) ocorreu um aumento na cobertura da espécie Ipomoea pes-caprae na área em que esta foi plantada com raiz. Podemos concluir que o melhor método de plantio da Ipomoea pes-caprea foi de mudas por enraizamento, e o aumento da mortalidade no mês novembro está relacionada a fatores antrópicos. Palavras-chave: Restinga, Recuperação da vegetação, Praia de Grumari.
\end{abstract}

\section{Recovery of beach vegetation - Grumari Beach, RJ}

\begin{abstract}
This work aims to evaluate methods for frontal dune restoration by comparing two techniques of planting Ipomoea pes-caprae species: propagation of cuttings and planting of seedlings in the protected area of Parque Natural Municipal de Grumari, RJ. They were planted 900 specimens of Ipomoea pes-capreae transplanted from the Restinga garden in August 2015, distributed equally between the two types of planting: 450 seedlings planted directly and 450 by propagating stem cuttings. In September (month 1) and October (month 2) 2015, it was observed that mortality was approximately $20 \%$ for planting rooted seedlings and $70 \%$ for planting by cutting. In November (month 3 ) it was observed mortality of $60 \%$ in the planting of seedlings by rooting and $90 \%$ of planting by cuttings. In January (month 5), there was an increase in the coverage of Ipomoea pes-caprae species on the area where it has been planted with root. We can conclude that the best planting method for Ipomoea pes-caprea was the seedlings rooting. The I. pes-caprae increased mortality in November was related to anthropogenic factors.
\end{abstract}

Keywords: Restinga, Plant recovery, Grumari beach. 


\section{Restauración de la vegetación de playa - Playa Grumari, RJ}

Resumen: Este estudio tiene como objetivo evaluar dos técnicas de plantación de la especie Ipomoea pes-Caprae (Convolvulaceae), propagación y siembra de plántulas de corte en un área de Protección del Medio Ambiente, Natural Municipal Parque Grumari, RJ. Ellos se plantaron 900 especies de Ipomoea pes-Caprea trasplantaron el jardín Restinga en agosto de 2015, distribuido en partes iguales entre los dos tipos de siembra: 450 plántulas sembradas directamente y 450 mediante la propagación de esquejes de tallos. En septiembre (mes 1) y octubre (mes 2 ) de 2015, se observó que la mortalidad fue de aproximadamente el $20 \%$ en la siembra de plántulas por el enraizamiento y 70\% la siembra por estaquias. Ya en noviembre (mes 3) se observó una mortalidad del 60\% en la siembra de plántulas por el enraizamiento y el $90 \%$ de la plantación por estacas. A partir de enero (mês 5) se produjo un aumento en la cobertura de la especie Ipomoea pes-caprae en la zona donde se sembró con la raíz. Podemos concluir que el mejor método de siembra Ipomoea pes-Caprea estaba a favor de las plántulas, y el aumento de la mortalidad en el mes en noviembre se relaciona con factores antropogénicos.

Palabras clave: Restinga, Recuperación de las plantas, Playa Grumari.

\section{INTRODUÇÃO}

As dunas frontais representam o limite entre 0 ambiente praial e as dunas interiores. São feições naturais da maioria das praias arenosas e recebem contínuo aporte de sedimentos, transportados pelos ventos dominantes (DE OLIVEIRA et al., 2007; ALMEIDA e SUGUIO, 2012). Essas desempenham um papel importante na manutenção e preservação da integridade da morfologia da costa, atuando como barreiras dinâmicas contra a ação de ondas e tempestades (CORDAZZ0 e SEELIGER, 1995).

As dunas costeiras são constituídas, do ponto de vista sedimentar, por acúmulo de areia fina dominante. A forma, o tamanho e orientação para cada setor de praia variam em função da direção, velocidade do vento dominante e tamanho das partículas (PEIX0T0, 2010). Sobre o substrato arenoso se estabelece um grupo de plantas característico, cuja distribuição obedece a um conjunto de fatores físicos que inclui a ação dos ventos, ondas e marés (MORENOCASASOLA, 1982; HESP, 1989).

As praias e dunas possuem seu próprio valor intrínseco, mas também fornecem muitos bens e serviços que beneficiam direta e indiretamente 0 homem. 0 ritmo crescente de alterações humanas nestes ambientes e o potencial humano para reconstruir a natureza de modo a prover serviços e funções ecológicas exigem que as atividades antrópicas sejam reconsideradas de muitas maneiras de torná-las compatíveis com a natureza (NORDSTROM, 2008). 
Os fatores condicionantes do processo de vulnerabilidade dunar, se associam tanto a fatores de caráter antrópico quanto relacionados à dinâmica costeira atuante no local, sendo exemplos: a presença e o estado de danificação da vegetação, a velocidade e direção dos ventos, a variação sazonal das chuvas, o trânsito de pessoas e veículos, edificações sobre a linha de costa e na zona de acumulação praial, entre outros (0LIVEIRA et al., 2004).

Segundo Muehe et al. (2010), em seus estudos na Praia do Peró, sugere que é fundamental a manutenção da cobertura vegetal das dunas para inibir o transporte de sedimento e também verificou que o padrão de sazonalidade das plantas reflete no transporte de sedimento, com 0 aumento da cobertura vegetal diminui o transporte de sedimento.

Em áreas urbanas, a pressão antrópica pode perturbar esses ecossistemas. A praia e a duna em desequilíbrio tornam-se uma ameaça para homem e para seus patrimônios materiais (LEITE e ANDRADE, 2004). Com intuito de recuperar estes ambientes em desequilíbrio algumas estratégias de recuperação podem ser realizadas.

Nordstrom (2008) sugere que sejam utilizadas cercas e o plantio de espécies vegetais para recuperação de dunas frontais. Com isto a deposição de sedimento eólico irá ocorrer de forma natural.

Menezes e Araújo (1999) mencionaram que a distribuição das espécies vegetais próximas ao mar está diretamente ligada com os processos dinâmicos que determinam o perfil praial, como a ação das ondas na linha de costa, a largura do pós-praia e a deposição de sedimentos. Espera-se que em períodos com acréscimo de sedimento ocorra uma expansão da vegetação da duna frontal e em períodos erosivos, ocorra um decréscimo da cobertura vegetal (CASTELLANI e SANTOS, 2000; HESP, 2000; PEIX0T0, 2010).

São vários os fatores que interferem na dinâmica da vegetação de dunas. As peculiaridades dos ambientes litorâneos promovem grande diversificação de formas e adaptações da vegetação, necessárias para sua permanência neste ecossistema. A ação das águas marinhas e dos ventos, as condições do solo e topografia do terreno constituem características marcantes das regiões litorâneas (ROMARIZ, 1996).

Estudos como os de Cordazzo e Costa (1989); Zamith e Dalmaso (2000); Scherer-Widmer (2001), Scherer et al. (2005); Zamith e Scarano (2006); e Martins et al. (2008) alertam quanto ao alto grau de degradação sofrido pelas restingas ao longo de toda costa brasileira e do mundo. 
Sendo, as restingas do Sul e sudeste brasileiro as formações vegetacionais mais destruídas e ameaçadas. Dentre os tipos fitofisionômicos das restingas estão incluídas, a restinga herbáceosubarbustiva de praias e dunas frontais (FALKENBERG, 1999).

Segundo Balensiefer (1997), a recuperação de áreas degradadas no Brasil é uma atividade relativamente recente, onde as técnicas empregadas parecem evoluir positivamente. Inicialmente as atividades de recuperação restringiam-se ao simples plantio de árvores (revegetação) e, atualmente, constituem-se em programas de recuperação ambiental.

0 Sistema Nacional de Unidades de Conservação da Natureza instituiu que o termo restauração compreende a restituição de um ecossistema ou de uma população silvestre degradada para o mais próximo possível de sua condição original (Lei n. ${ }^{\circ}$ 9.985/2000, art. $2^{\circ}$, inciso XIV). Restauração refere-se ao conjunto de tratamentos que visa recuperar a forma original do ecossistema, ou seja, a sua estrutura original, dinâmica e interações biológicas, sendo geralmente recomendada para ecossistemas raros e ameaçados, pois demanda mais tempo e os custos são significativamente maiores (JESUS, 1997).

Este trabalho visa contribuir com a integridade da duna frontal, a título de restaurar este ambiente, avaliando duas técnicas de plantio da espécie Ipomoea pes-caprae: propagação de estaquia e plantio por mudas, na Área de Proteção Ambiental (APA) do Parque Natural Municipal de Grumari, RJ.

\section{MATERIAL E MÉTODOS}

\section{1. Área de Estudo}

A Praia de Grumari está localizada no bairro de mesmo nome, na Região Metropolitana do município do Rio de Janeiro. Grumari possui uma área de 966,32 hectares e de acordo com Silva et al. (2016) as áreas de restingas, brejos, praias e costões rochosos de Grumari correspondem a 15\% do bairro, dos quais 10\% referem-se à vegetação de restinga.

Na década de 80, a praia de Grumari foi tombada na categoria de Monumento Natural, sob a tutela do Instituto Estadual do Patrimônio Artístico e Cultural (Inepac), esta medida buscou reconhecer os valores paisagísticos do local, além disso, condicionou quaisquer intervenções (urbanas) na área à aprovação do Inepac (SILVA et al., 2016). 
0 Decreto Lei Municipal No 944 de 30 de dezembro de 1986 determinou a criação da Área de Proteção Ambiental (APA) de Grumari, esta categoria de Unidade de Conservação tem como objetivos básicos proteger a diversidade biológica, disciplinar o processo de ocupação e assegurar a sustentabilidade do uso dos recursos naturais. A APA de Grumari possui áreas sobrepostas ao Parque Estadual da Pedra Branca (acima da cota $100 \mathrm{~m}$ de altitude) e à APA do Maciço da Pedra Branca (acima da cota 300 m) (FUNBI0; SMAC, 2012). Portanto, este bairro representa uma amostra da paisagem natural carioca de outrora e é legalmente protegido por unidades de conservação.

Segundo a classificação de Koppen, o clima do Parque Natural Municipal de Grumari está inserida na região delimitada como subtipo Aw, clima tropical com uma estação seca em que a precipitação média mensal é inferior a $60 \mathrm{~mm}$ em pelo menos um mês por ano, na época de Sol mais baixo e dias mais curtos (inverno). 0 Parque está situado na faixa de precipitação de 1.001 a $1.200 \mathrm{~mm}$ nas suas partes sudoeste e leste e de 1.201 a $1.400 \mathrm{~mm}$ na sua parte central e áreas elevadas, com uma insolação na faixa entre $1.800 \mathrm{~h}$ e $2.000 \mathrm{~h}$ por ano. As temperaturas médias mínimas variam entre $18^{\circ} \mathrm{C}$ e $20^{\circ} \mathrm{C}$, enquanto as temperaturas médias máximas situam-se entre $27^{\circ} \mathrm{C}$ e $29^{\circ} \mathrm{C}$, com temperaturas médias anuais entre $22^{\circ} \mathrm{C}$ e $24^{\circ} \mathrm{C}$. De acordo com o Plano de Manejo de Parque Natural Municipal de Grumari (2012), ocorrem argissolos na maior parte da área da unidade e praias que representam $4,72 \%$ da área de estudo. Destaca-se que área tem baixa diversidade geológica, composta por gnaisses da Unidade Rio Negro, granitos indiferenciados da Unidade Rochas Graníticas e depósitos recentes da Unidade Sedimentos Quaternários.

\section{Levantamento Florístico}

Foi realizado um levantamento fitossociológico na vegetação de praia, na praia de Grumari com intuito de maior conhecimento desta vegetação. 0 levantamento florístico e fitossociológico da vegetação foi realizado no mês de julho de 2015 por meio do método visual. Os espécimes registrados foram devidamente identificados de acordo com suas famílias botânicas e nomes científicos, para isto utilizou-se a Lista de Espécies da Flora do Brasil elaborada pelo Jardim Botânico do Rio de Janeiro (2016). 
Foram verificadas a Cobertura Absoluta, Cobertura Relativa, Frequência Absoluta, Frequência Relativa, Valor de Importância e Índice de Diversidade em três pontos da praia de Grumari (P1- oeste da praia, P2- central da praia, P3- leste da praia). Foram realizados cálculos de Índice de Diversidade de Shannon (H’) para descrever a estrutura da comunidade vegetal (BROWER e ZAR, 1977). Estes cálculos serão feitos com base nos valores médios de cobertura vegetal de cada espécie, amostradas em cada estação do ano.

$$
\begin{aligned}
& \mathrm{H}^{\prime}=\left(\mathrm{Pi}^{*} \log \mathrm{Pi}\right) \text { e } \mathrm{J}=\mathrm{H}^{\prime} / \log \mathrm{S} \\
& \mathrm{Pi} \text { = valor de cobertura média das espécies/valor de cobertura média de todas as espécies } \\
& \mathrm{S}=\text { Riqueza de espécies }
\end{aligned}
$$

\section{Escolha da espécie e Plantio de mudas}

A espécie Ipomea pes-capreae da família Convolvulaceae foi escolhida para ser plantada na área de estudo por ser considerada uma planta pioneira, típica de dunas, com distribuição pantropical, que apresenta ramificações que tendem a fixar o pacote arenoso sob a área na qual se estabelece (GOMES-NET0, 2004). Uma espécie muito encontrada extensivamente sobre as dunas das praias em toda região litorânea brasileira, principalmente na porção frontal das dunas e apresenta um grande potencial para utilização em projetos de reabilitação de dunas, com base nestas características definiu-se analisar o plantio desta espécie.

Foram plantados 900 espécimes de Ipomea pes-capreae, transplantadas do horto Restinga, em agosto de 2015, distribuídas equitativamente entre os dois tipos de plantio: 450 mudas diretamente plantadas e 450 por propagação de estaquia. 0 plantio foi realizado por meio de abertura de covas na areia, nas quais os fragmentos foram preparados com distância de $5 \mathrm{~cm}$, dispostos em 30 quadradros de $1 \mathrm{~m}^{2}$. Em cada cova foi adicionado hidrogel com distância de $0,5 \mathrm{~m}$ entre eles ${ }^{1}$. As áreas foram delimitadas com estacas de madeira e os pontos marcados com GPS (Sistema de Posicionamento Global, Garmim Oregon 550). A quantidade de lixo foi

\footnotetext{
${ }^{1}$ Polímero de alta qualidade e pureza, que absorve de 200 a 400 vezes seu peso em água e chega a aumentar em 100 vezes seu tamanho (Forth Hidrogel).
} 
estimada por peso, mensalmente, utilizando-se piezômetro conforme procedimento descrito em Rosa e Cordazzo (2007).

\section{Granulometria}

Para acompanhar o comportamento da evolução do plantio na sua relação com a distribuição granulométrica foram coletados sedimentos nos setores da face praial (a face praial localiza-se na zona intermareal, onde geralmente ocorrem os processos de espraiamento das ondas fluxo e refluxo), pós-praia (sedimentos do pós-praia, transportados pela ação do vento, são os principais responsáveis pela formação e desenvolvimento da duna frontal), duna frontal (herbácea) e duna frontal (arbustiva) (estabelece o limite entre a praia e os demais ambientes da zona costeira). Os sedimentos coletados foram submetidos à análise granulométrica no Laboratório de Geografia Física da UFF. 0 processamento dos dados granulométricos foi realizado através do programa de análise Sysgran 3.1, baseados na classificação granulométrica de acordo com a escala de Wentworth (1922).

Como medida do controle do sucesso do plantio foi retirada a vegetação exótica encontrada na área de estudo. Em paralelo procedeu-se à observação das taxas de mortalidade das espécies plantadas com contagem mensal dos indivíduos. 0 crescimento das mudas foi medido pelo aumento do percentual de cobertura da vegetação na malha amostral.

0 acompanhamento da ocorrência de outras espécies foi realizado com amostragens mensais da vegetação em quadrados permanentes de $1 \mathrm{~m}^{2}$ (marcados com estacas de madeira). Em cada quadrado foi avaliada a ocorrência e a porcentagem da cobertura vegetal de cada espécie. A porcentagem de cobertura vegetal foi estimada visualmente e, para isto, o quadrado foi subdividido em quatro partes para auxiliar nas avaliações (MÜELLER-DOMBOIS e ELLEMBERG, 1974).

Para análise da precipitação, pluviosidade e cobertura vegetal foi utilizado Análise de Componentes Principais - ACP (LANDIM, 2000). 


\section{RESULTADOS E DISCUSSÃ0}

\section{Levantamento florístico}

Nas análises de cobertura vegetal dos três setores estudados (P1 a oeste, P2 central e P3 a leste) na Praia de Grumari, registrou-se um total de 17 famílias botânicas, 31 espécies, sendo que 12 espécies de porte arbustivo. As espécies Ipomoea imperati, Ipomoea pes-caprae, Sophora tomentosa e a Poaceae Indeterminada GI foram encontradas nos três setores. Destaca-se a família Fabaceae como a de maior representatividade na área (Tabela 1).

Tabela 1 - Lista florística das espécies encontradas nos três setores estudados na praia de Grumari, Rio de Janeiro/RJ

\begin{tabular}{|c|c|c|c|}
\hline Família/Espécie & $\mathrm{Pl}$ & $\mathrm{P} 2$ & P3 \\
\hline \multicolumn{4}{|l|}{ ARECACEAE } \\
\hline Allagoptera arenaria (Gomes) Kuntze. & $\mathrm{X}$ & & $\mathrm{X}$ \\
\hline \multicolumn{4}{|l|}{ ANACARDIACEAE } \\
\hline Schinus terebinthifolia Raddi & & & X \\
\hline \multicolumn{4}{|l|}{ AMARANTHACEAE } \\
\hline Alternanthera maritima (Mart.) St.-Hil. & $\mathrm{X}$ & $\mathrm{X}$ & \\
\hline \multicolumn{4}{|l|}{ ARALIACEAE } \\
\hline Hydrocotyle boranienses Lam & & & $\mathrm{X}$ \\
\hline \multicolumn{4}{|l|}{ ASTERACEAE } \\
\hline Mikania cordifolia (L.f.) Wild & & & $\mathrm{X}$ \\
\hline \multicolumn{4}{|l|}{ BIGNONIACEAE } \\
\hline Arrabidea conjugata (Vell.) Mart & & & $\mathrm{X}$ \\
\hline \multicolumn{4}{|l|}{ CLUSIACEAE } \\
\hline Clusia fluminensis Planch. e Triana & & & $\mathrm{X}$ \\
\hline \multicolumn{4}{|l|}{ CACTACEAE } \\
\hline $\begin{array}{l}\text { Opuntia arechevaletai Spegazzini ex } \\
\text { Arechevaleta }\end{array}$ & \multicolumn{2}{|c|}{ Arechevaleta } & \\
\hline Cereus fernambucensis Lem. & & & $X$ \\
\hline Pilosocereus arrabidae (Lem.) Byles & & & $\mathrm{X}$ \\
\hline \multicolumn{4}{|l|}{ CONVOLVULACEAE } \\
\hline Ipomoea imperati (Valhl) Griseg & $\mathrm{X}$ & $X$ & $\mathrm{X}$ \\
\hline Ipomoea pes-caprae (L.) R. Brown & $\mathrm{X}$ & $X$ & $\mathrm{X}$ \\
\hline \multicolumn{4}{|l|}{ CYPERACEAE } \\
\hline Remirea maritima Aubl & & & X \\
\hline
\end{tabular}




\begin{tabular}{|c|c|c|c|}
\hline Família/Espécie & P1 & P2 & P3 \\
\hline \multicolumn{4}{|l|}{ EUPHORBIACEAE } \\
\hline Sebastiania corniculata (Vahl.) Muell. Arg. & & $\mathrm{X}$ & \\
\hline \multicolumn{4}{|l|}{ FABACEAE } \\
\hline Canavalia rosea (Sw.) & & $\mathrm{X}$ & $\mathrm{X}$ \\
\hline Dalbergia ecastaphyllum (L.) Taub. & $X$ & & \\
\hline Senegalia bonariensis (Gillies ex Hook e Arn.) & & & $\mathrm{X}$ \\
\hline \multicolumn{4}{|l|}{ Seigler e Ebinger } \\
\hline Sophora tomentosa L. & $X$ & $\mathrm{X}$ & $\mathrm{X}$ \\
\hline Pitheceolobium tortum Mart. & & $X$ & \\
\hline Chamaecrista ensiformis (Vell.) & & $\mathrm{X}$ & \\
\hline \multicolumn{4}{|l|}{ MALPIGHIACEAE } \\
\hline Heteropterys chrysophylla (Lam) DC & & & $\mathrm{X}$ \\
\hline \multicolumn{4}{|l|}{ MYRTACEAE } \\
\hline Eugenia uniflora L. & & & X \\
\hline Myrrhinium atropurpureum Schott. & & $\mathrm{X}$ & \\
\hline \multicolumn{4}{|l|}{ POACEAE } \\
\hline Panicum racemosum (P. Beauv.) Spreng. & & $\mathrm{X}$ & $\mathrm{X}$ \\
\hline Paspalum vaginatum Sw. & X & & $X$ \\
\hline indeterminada (Gr.I) & $\mathrm{X}$ & $X$ & X \\
\hline Indeterminada (Gr. II) & & $\mathrm{X}$ & $\mathrm{X}$ \\
\hline \multicolumn{4}{|l|}{ SAPOTACEAE } \\
\hline Manilkara subsericea (Mart) Dubard & & $X$ & \\
\hline
\end{tabular}

Fonte: Própria.

Cabe destacar que Silva et al. (2016) realizou um levantamento bibliográfico durante o ano de 2014 e listou 41 espécies no Parque Municipal de Grumari, sendo que destas, somente nove espécies foram observadas neste trabalho, logo, as demais 22 espécies encontradas, ainda não foram registradas nas bibliografias consultadas.

Pode-se observar que o Pl (setor oeste) apresentou o menor número de espécie (10) assim como o menor índice de diversidade (H’) (tabela 2). A espécie com maior valor de importância foi a arbustiva Dalbergia ecastaphyllum (Tabela 2). No P2 (setor central) foram observadas 14 espécies e o maior valor de importância (VI) foi de uma espécie arbustiva: Sophora tomentosa (tabela 3). No P3 (setor leste) apresentou o maior número de espécies (20) e 0 maior índice de diversidade (H’). E o maior valor de importância foi da Myrtaceae, Eugenia uniflora. 
Tabela 2 - Parâmetros fitossociológicos das espécies amostradas no Pl (setor Oeste) da praia de Grumari, Rio de Janeiro/RJ. (CA= Cobertura Absoluta, $\mathrm{CR}=$ Cobertura Relativa, $\mathrm{FA}=$ Frequência Absoluta, FR= Frequência Relativa, Valor de Importância, H'= índice de diversidade)

\begin{tabular}{lcccccc}
\hline \multicolumn{1}{c}{ PI } & CA & CR & FA & FR & VI & H' \\
\hline Ipomoea imperati & 14,17 & 12,06 & 3 & 15 & 27,06 & $-0,11$ \\
Paspalum vaginatum & 15,00 & 12,77 & 3 & 15 & 27,77 & $-0,11$ \\
Alternanthera maritima & 0,83 & 0,71 & 1 & 5 & 5,71 & $-0,02$ \\
Ipomoea pes-Caprae & 0,83 & 0,71 & 1 & 5 & 5,71 & $-0,02$ \\
Allagoptera arenaria & 0,83 & 0,71 & 1 & 5 & 5,71 & $-0,02$ \\
Sophora tomentosa & 10,83 & 9,22 & 1 & 5 & 14,22 & $-0,10$ \\
Dalbergia ecastaphyllum & 27,50 & 23,40 & 4 & 20 & 43,40 & $-0,15$ \\
Opuntia arechevaletai & 5,00 & 4,26 & 1 & 5 & 9,26 & $-0,06$ \\
indeterminada (Gr.I) & 17,50 & 14,89 & 2 & 10 & 24,89 & $-0,12$ \\
Mikania cordifolia & 25,00 & 21,28 & 3 & 15 & 36,28 & $-0,14$ \\
\hline & 117,5 & $\mathbf{1 0 0}$ & 20 & $\mathbf{1 0 0}$ & 200 & $\mathbf{0 , 8 4}$ \\
\hline
\end{tabular}

Fonte: Própria.

Tabela 3 - Parâmetros fitossociológicos das espécies amostradas no P2 (setor Central) da praia de Grumari, Rio de Janeiro/RJ. (CA= Cobertura Absoluta, $\mathrm{CR}=$ Cobertura Relativa, FA= Frequência Absoluta, FR= Frequência Relativa, Valor de Importância, $H^{\prime}=$ indice de diversidade)

\begin{tabular}{lcccccc}
\hline \multicolumn{1}{c}{ P2 } & CA & CR & FA & FR & VI & H' \\
\hline Alternanthera maritima & 5,62 & 5,26 & 3 & 9,67 & 14,94 & $-0,06$ \\
Canavalia rósea & 15,62 & 14,61 & 3 & 9,67 & 24,29 & $-0,12$ \\
Chamaecrista ensiformis & 2,5 & 2,33 & 1 & 3,22 & 5,56 & $-0,03$ \\
indeterminada (Gr.I) & 16,87 & 15,78 & 4 & 12,90 & 28,69 & $-0,12$ \\
Indeterminada (Gr.II) & 3,75 & 3,50 & 5 & 16,12 & 19,63 & $-0,05$ \\
Ipomoea Imperati & 10 & 9,35 & 5 & 16,12 & 25,48 & $-0,09$ \\
Ipomoea pes-caprae & 3,75 & 3,50 & 1 & 3,22 & 6,73 & $-0,05$ \\
Manilkara subsericea & 1,25 & 1,16 & 1 & 3,22 & 4,39 & $-0,02$ \\
Myrrhinium & 0,62 & 0,58 & 1 & 3,22 & 3,81 & $-0,01$ \\
atropurpureum & & & & & & \\
Opuntia arechevaletai & 12,5 & 11,69 & 1 & 3,22 & 14,92 & $-0,10$ \\
Panicum racemosum & 1,25 & 1,16 & 1 & 3,22 & 4,39 & $-0,02$ \\
Pithecolobium tortum & 1,25 & 1,16 & 1 & 3,22 & 4,39 & $-0,02$ \\
Sebastiania corniculata & 6,25 & 5,84 & 2 & 6,45 & 12,29 & $-0,07$ \\
Sophora tomentosa & 25,62 & 23,97 & 2 & 6,45 & 30,42 & $-0,14$ \\
\hline & 106,87 & $\mathbf{1 0 0}$ & 31 & $\mathbf{1 0 0}$ & $\mathbf{2 0 0}$ & $\mathbf{0 , 9 6}$ \\
\hline
\end{tabular}

Fonte: Própria. 
Tabela 4 - Parâmetros fitossociológicos das espécies amostradas no P3 (setor Leste) da praia de Grumari, Rio de Janeiro/RJ. (CA= Cobertura Absoluta, $\mathrm{CR}=$ Cobertura Relativa, $\mathrm{FA}=$ Frequência Absoluta, FR= Frequência Relativa, Valor de Importância, H'= índice de diversidade)

\begin{tabular}{lcccccc}
\hline \multicolumn{1}{c}{ P3 } & CA & CR & FA & FR & VI & H' \\
\hline Allagoptera arenaria & 0,45 & 0,40 & 1,00 & 2,78 & 3,18 & $-0,01$ \\
Arrabidea conjugata & 7,27 & 6,48 & 2,00 & 5,56 & 12,03 & $-0,08$ \\
Canavalia rósea & 4,55 & 4,05 & 3 & 8,33 & 12,38 & $-0,06$ \\
Cereus fernambucensis & 3,64 & 3,24 & 2,00 & 5,56 & 8,79 & $-0,05$ \\
Clusia fluminensis & 3,64 & 3,24 & 1,00 & 2,78 & 6,02 & $-0,05$ \\
Eugenia uniflora & 25,45 & 22,67 & 3,00 & 8,33 & 31,01 & $-0,15$ \\
Heteropterys chrysophylla & 1,82 & 1,62 & 2,00 & 5,56 & 7,17 & $-0,03$ \\
Hydrocotyle boranienses & 0,91 & 0,81 & 1,00 & 2,78 & 3,59 & $-0,02$ \\
indeterminada (Gr.I) & 23,18 & 20,65 & 3 & 8,33 & 28,98 & $-0,14$ \\
Indeterminada (Gr.II) & 5,45 & 4,86 & 1 & 2,78 & 7,64 & $-0,06$ \\
Ipomoea imperati & 4,09 & 3,64 & 3 & 8,33 & 11,98 & $-0,05$ \\
Ipomoea pes-caprae & 10,00 & 8,91 & 4 & 11,11 & 20,02 & $-0,09$ \\
Panicum racemosum & 9,09 & 8,10 & 2 & 5,56 & 13,65 & $-0,09$ \\
Paspalum vaginatum & 0,91 & 0,81 & 1 & 2,78 & 3,59 & $-0,02$ \\
Pilosocereus arrabidae & 1,82 & 1,62 & 2,00 & 5,56 & 7,17 & $-0,03$ \\
Remirea marítima & 1,36 & 1,21 & 2 & 5,56 & 6,77 & $-0,02$ \\
Schinus terebinthifolia & 0,45 & 0,40 & 1,00 & 2,78 & 3,18 & $-0,01$ \\
Senegalia bonariensis & 3,64 & 3,24 & 1,00 & 2,78 & 6,02 & $-0,05$ \\
Sophora tomentosa & 4,55 & 4,05 & 1 & 2,78 & 6,83 & $-0,06$ \\
\hline & 112,27 & $\mathbf{1 0 0}$ & 36 & $\mathbf{1 0 0}$ & 200 & $\mathbf{1 , 0 5}$ \\
\hline
\end{tabular}

Fonte: Própria.

Foi observado em todos os setores que, próximo à Avenida Estado da Guanabara, ocorreu uma maior abundância da Poaceae Indeterminada (GrI), este espécime é semelhante ao capim gordura (Melinis minutiflora P. Beauv), sendo uma espécie invasora e exótica. A interferência antrópica causada pela inserção da estrada provoca a fragmentação do habitat, modifica a estrutura da paisagem, gerando mudanças na composição e na diversidade das comunidades adjacentes à borda. Essa interferência agiu fundamentalmente reduzindo e isolando as áreas propícias à sobrevivência das populações, resultando em uma variedade de efeitos físicos e biológicos. Essas alterações são conhecidas como efeito de borda. 


\section{Plantio e monitoramento Ipomoea pes-caprae}

A título de proteção do plantio foi introduzida uma cerca utilizando-se madeira de Pinus tratado, cedido pela Prefeitura do Rio de Janeiro. Nos meses de setembro (mês 1) e outubro (mês 2) de 2015 , observou-se que a mortalidade foi de aproximadamente $20 \%$ para o plantio de mudas enraizadas e 70\% para o plantio por estaquias. De acordo com Gomes-Neto et al. (2004), a maior taxa de mortalidade ocorre nas primeiras semanas, como consequência do estresse que as estacas e mudas sofrem ao serem plantadas na duna, a baixa capacidade de retenção de água pelo sedimento e às altas temperaturas que ocorrem nas dunas frontais. Tais fatores fazem com que as plantas consumam parte de suas reservas energéticas para resistir a essas adversidades, reduzindo a quantidade de energia alocada para o crescimento, logo, diminuindo as chances de sucesso do estabelecimento em campo.

No mês de novembro (mês 3) observou-se uma mortalidade de 60\% no plantio de mudas por enraizamento e 90\% do plantio por estaquia (Figura 1). Esperava-se uma redução na taxa de mortalidade neste mês, no entanto, este aumento abrupto pode ter sido ocasionado pelo uso público da praia de Grumari, que no mês de novembro recebeu um número maior de banhistas devido a proximidade com a verão. Muitos banhistas utilizaram a área de plantio para fazer churrasco, o que não é permitido nas praias do município do Rio de Janeiro, com isso várias mudas de Ipomoea pes-caprae acabam sendo queimadas. Em dezembro também foi observado uma elevada mortalidade, de quase $70 \%$ nas mudas enraizadas.

Após o ocorrido foi colado arame nas estacas de madeira com intuito de tentar reduzir o impacto na área de plantio e uma placa de sinalização. No mês de janeiro (mês 5) parte da cerca foi cortada e foram retirados quatro mourões da cerca (Fotos 1 a 4). A partir de dezembro (mês 4) ocorreu um aumento na cobertura da espécie Ipomoea pes-caprae na área em que esta foi plantada com raiz (Figura 2).

$\mathrm{Na}$ área em que foram plantadas as estaquias Ipomoea pes-caprae não se observou expansão da cobertura da Ipomoea pes-caprae. Outras espécies foram verificadas, tais como Ipomoea imperati, Remirea maritima e Paspalum vaginatum. As espécies tiveram um aumento de cobertura vegetal nos meses de janeiro e fevereiro. 


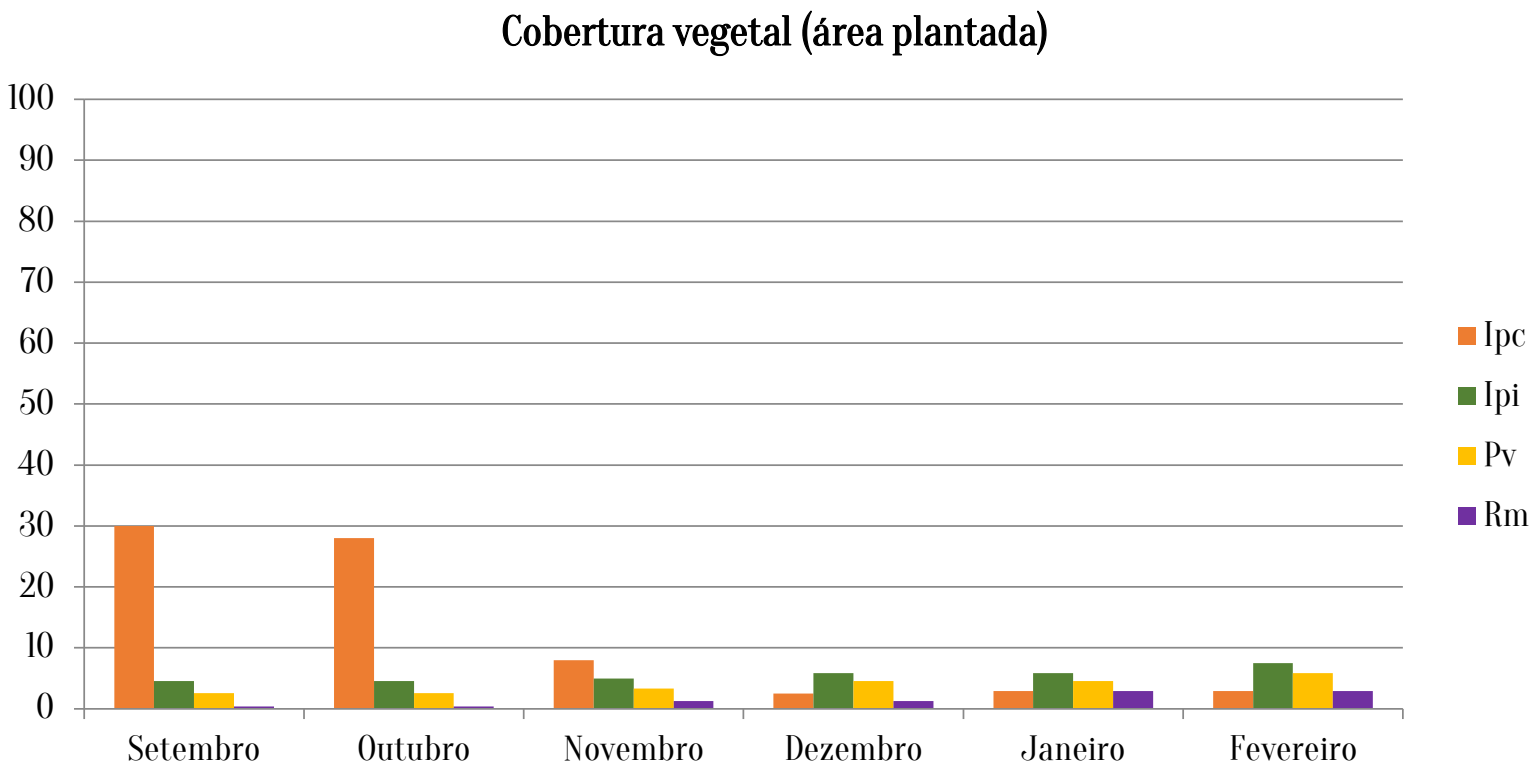

Figura 1. Cobertura vegetal na área da vegetação plantada com estaquias. Legenda: Ipc: Ipomoea pes-caprae; Ipi: Ipomoea imperati, Rm: Remirea marítima; Pv: Paspalum vaginatum

Fonte: Própria.

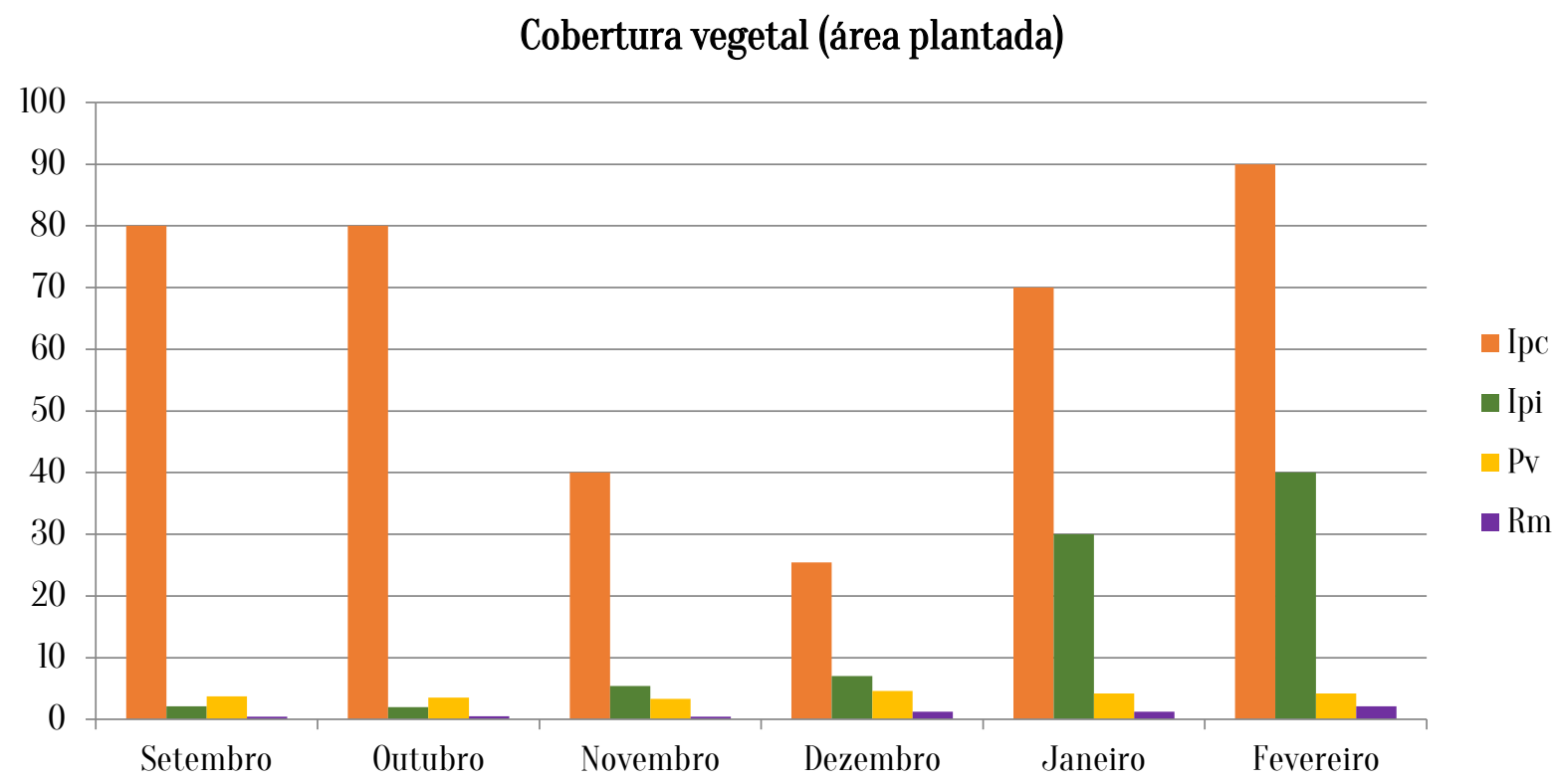

Figura 2. Cobertura vegetal na área da vegetação plantada com enraizamento. Legenda: Ipc: Ipomoea pes-caprae; Ipi: Ipomoea imperati, Rm: Remirea marítima; Pv: Paspalum vaginatum

Fonte: Própria. 


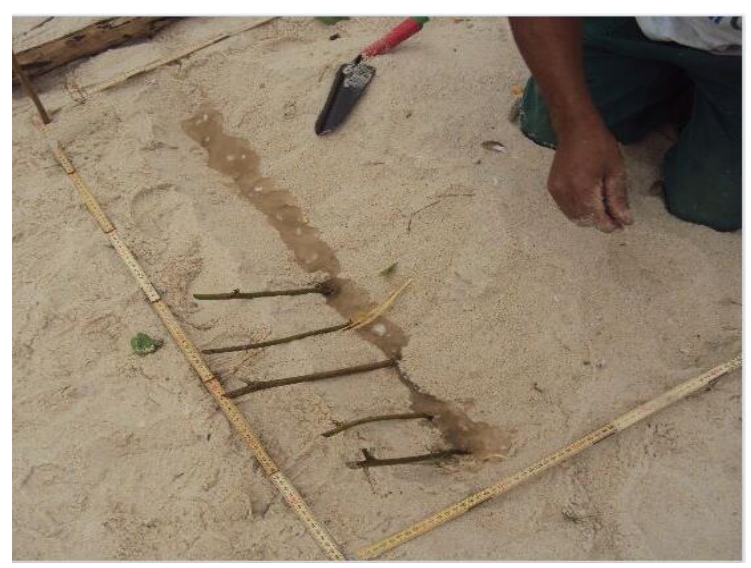

Foto 1. Plantio de Ipomoea pes-caprae por estaquias com hidrogel

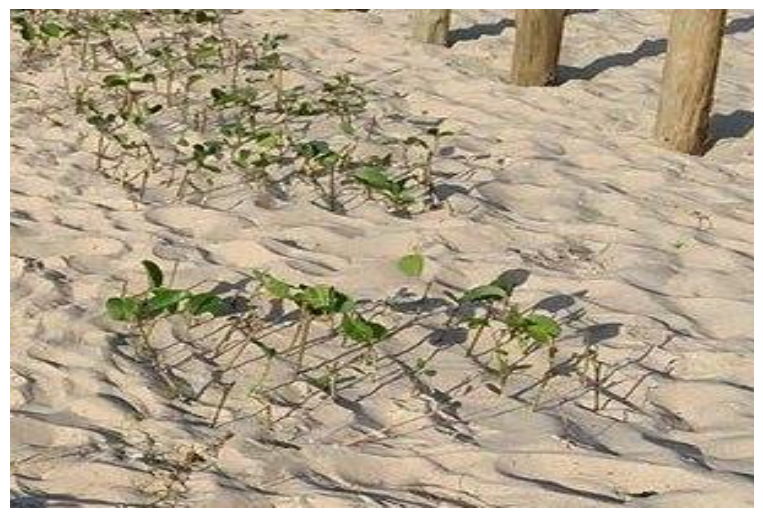

Foto 3. Plantio de Ipomoea enraizada

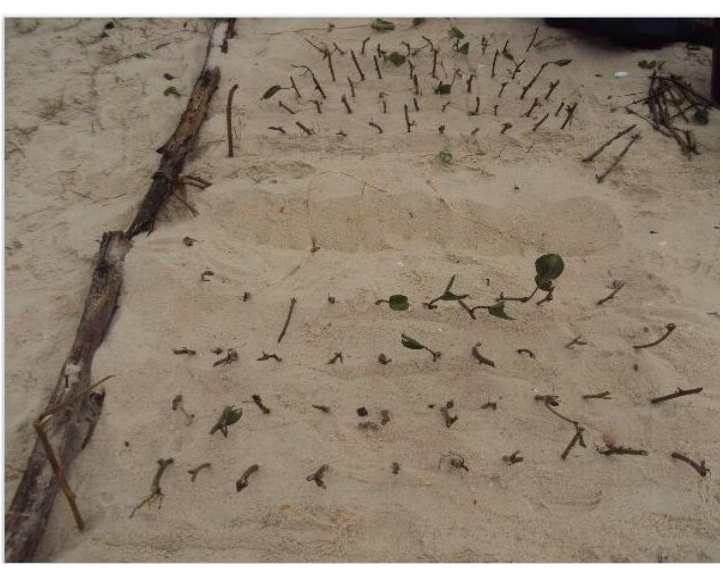

Foto 2. Plantio de Ipomoea pes-caprae por estaquias

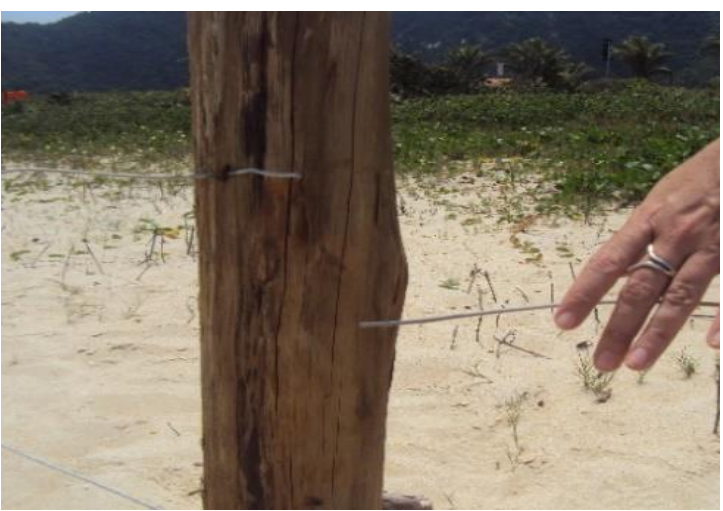

Foto 4. Cerca cortada

\section{Granulometria}

Segundo Santos (2010), o estágio morfodinâmico da praia de Grumari foi classificado como reflectivo. As praias reflectivas ocorrem em ambientes que combinam baixa energia de onda, longo período e grãos grossos e pequenas dunas frontais (SH0RT, 1999). A análise da granulometria realizada na área de estudo mostrou a predominância de grãos grossos nos quatro pontos coletados, com uma pequena variação de grãos mais finos na duna herbácea e arbustiva (Figura 3), corroborando o observado por Santos (2010). 

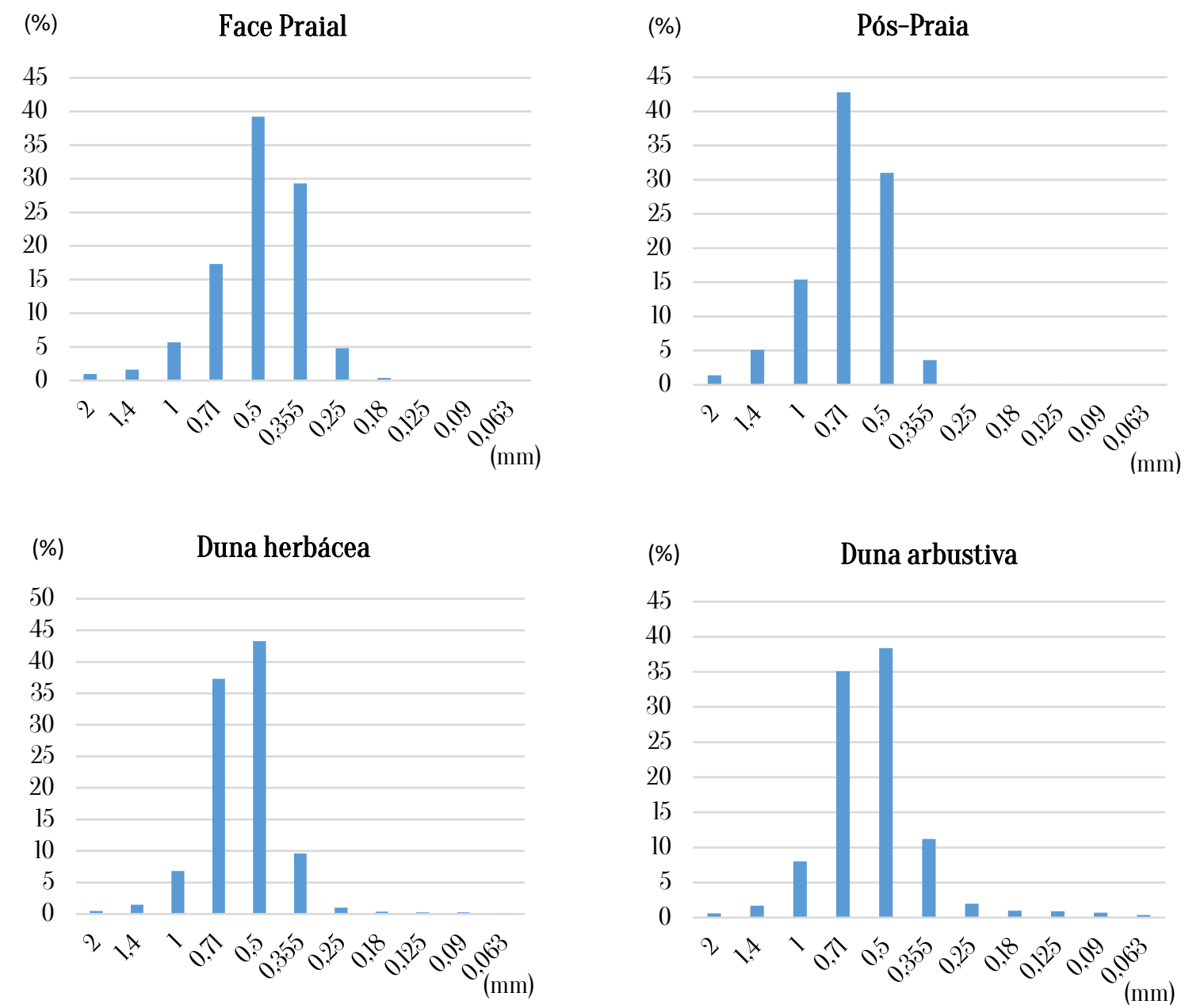

Figura 3. Percentual de grãos (\%) por diâmetro $(\mathrm{mm})$ em cada ponto amostral da área de estudo, Praia de Grumari, Rio de Janeiro/RJ, julho/2015

Fonte: Própria.

\section{Retirada da vegetação exótica e de lixo da Praia de Grumari}

A retirada da vegetação exótica foi realizada em uma área de $117 \mathrm{~m}^{2}$. Foram retiradas 2 espécies exóticas. Uma espécie é a Agave americana L. da família Amaryllidacea, comumente conhecida como piteira, originária do México e das Antilhas. A outra espécie, Terminalia catappa L. da família Combretaceae, comumente conhecida como castanheira ou sombreiro, esta espécie é nativa da Índia. Foram retirados dois indivíduos da área de estudo. No local onde foi retirada uma Terminalia catappa com cerca de 2,40m de altura e sua raiz com $2 \mathrm{~m}$ de diâmetro. Após a retirada desta espécie foram plantados dois indivíduos da espécie Eugenia uniflora (pitangueira). 
Quanto ao lixo coletado na área de estudo, obteve-se uma média mensal de 15kg, em que se encontrou as maiores quantidades de resíduos sólidos nos meses de verão como Dezembro, Janeiro e Fevereiro. Grande parte do lixo era composto por materiais plásticos como garrafas PET e fragmentos de sacolas, garrafas de vidro e latas de alumínio. Outros estudos mostram que nas praias, o padrão mundial é apresentar uma maior porcentagem de plástico em relação a outros itens do lixo (MADZENA e LASIAK, 1997; MOORE et al., 2001; ARAÚJ0 e COSTA, 2004; MASCARENHAS et al., 2008). Tal afirmação foi observada na coleta de lixo deste estudo, mas é importante destacar que na área em análise as garrafas de vidro e as latas de alumínio também representavam uma grande porcentagem do total de lixo observado. A elevada quantidade de lixo encontrada mensalmente evidenciou as atividades antrópicas que aconteciam na área.

\section{Análise de Componentes Principais - ACP}

De acordo com o Plano de Manejo de Parque Natural Municipal de Grumari (2012), a área de estudo apresenta precipitação média total anual entre 1.000 a $1.200 \mathrm{~m}$. As temperaturas médias mínimas variam entre $18^{\circ} \mathrm{C}$ e $20^{\circ} \mathrm{C}$, enquanto as temperaturas médias máximas situamse entre $27^{\circ} \mathrm{C}$ e $29^{\circ} \mathrm{C}$, com temperaturas médias anuais entre $22^{\circ} \mathrm{C}$ e $24^{\circ} \mathrm{C}$.

Para analisar os dados de precipitação, temperatura e cobertura vegetal estes dados foram correlacionados em um ACP, onde o eixo 1 (51,17\%) e eixo 2 (18,16\%) acumularam 69,33\% do total da variância. Pode-se verificar que as maiores correlações foram no eixo 2, entre a cobertura vegetal da Ipomoea pes-caprae e a precipitação.

Estudos indicam que o crescimento vegetativo da Ipomoea pes-caprae está relacionado com períodos quentes, úmidos e de maior precipitação (CORDAZZ0 e SOUZA, 1984; BERNARDI e SEELIGER, 1989; COSTA e SEELIGER, 1990; CASTELLANI, 2003, PEIXOTO, 2010).

Zamith e Scarano (2006) sugerem que o plantio direto de mudas é a melhor estratégia para o plantio de vegetação de duna, uma vez que o estabelecimento de plântulas a partir de sementes é muito difícil no substrato arenoso da restinga, pobre em nutrientes, com alta insolação e baixa retenção hídrica. 0 trabalho realizado por Gome-Neto et al. (2004) vem a corroborar com os resultados verificados até o presente momento, onde o processo de enraizamento prévio em viveiro favoreceu a sobrevivência das mudas, resultando numa 
diferença positiva no plantio da espécie Ipomoea pes-caprae. Cabe ressaltar que o plantio realizado na praia de Grumari foi sem irrigação.

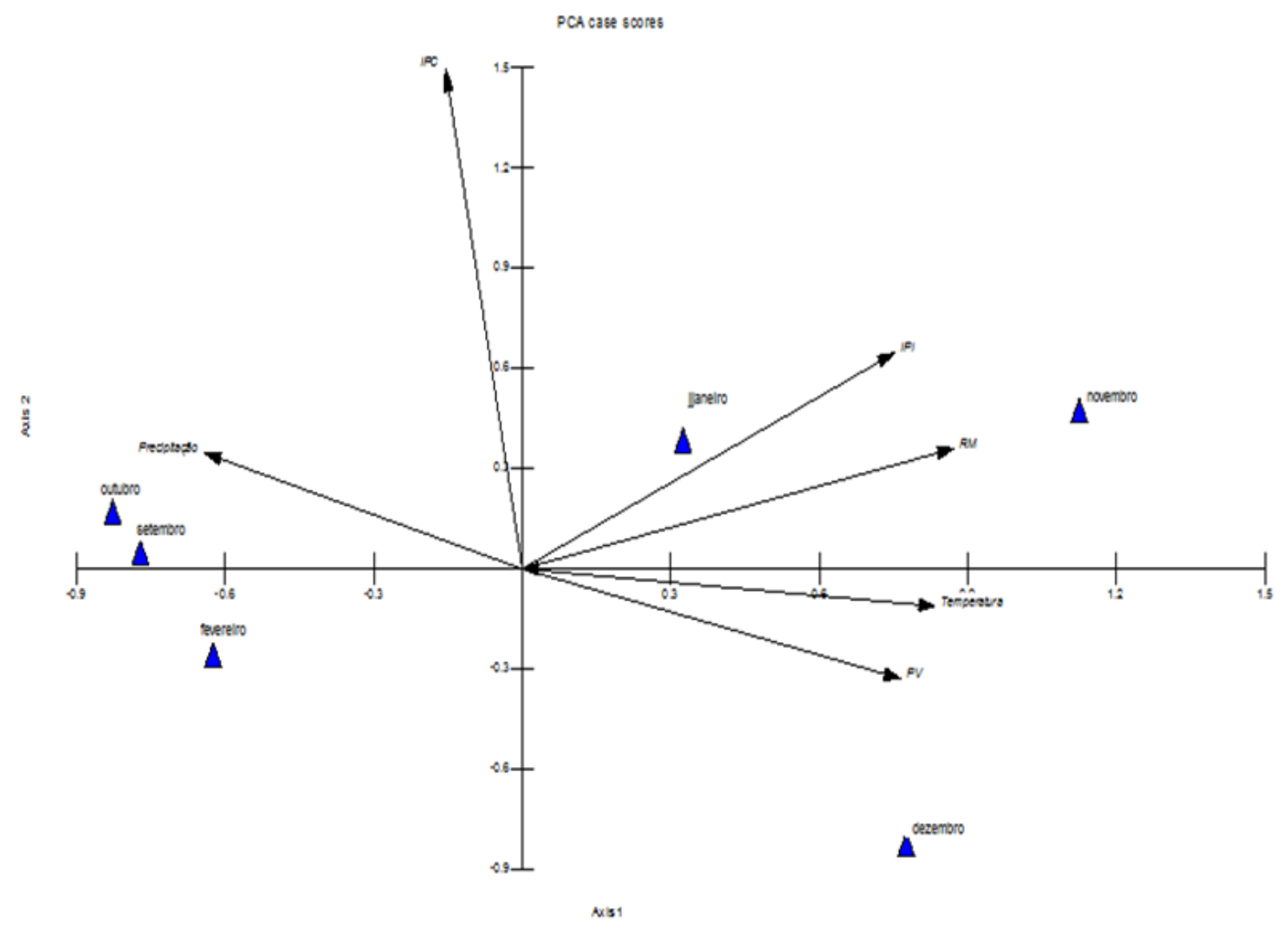

Figura 4. Análise de componentes principais (ACP), realizado com distância euclidiana para os dados de precipitação, temperatura e cobertura vegetal

Fonte: Própria.

\section{CONCLUSÃ0}

Podemos concluir que na área de estudo havia um total de 31 espécies botânicas e com relação ao plantio da espécie Ipomoea pes-caprea, pode-se afirmar que o método de mudas por enraizamento foi mais satisfatório, visto que este apresentou menor taxa de mortalidade. Entretanto, é importante destacar que ocorreu um aumento da mortalidade da espécie nos dois métodos avaliados, nos meses de novembro e dezembro de 2015. Tal fato pode estar relacionado ao fator antrópico, em função da maior presença de banhistas nestes meses, mas seria necessária uma análise mais aprimorada para definir se as ações antrópicas foram determinantes no aumento da taxa de mortalidade. Este trabalho está contribuindo 
efetivamente com a gestão do Parque Natural Municipal de Grumari, que tem uma enorme carência de pessoas que possam fazer a manutenção do equilíbrio e sustentabilidade ecológica, prestando assim um serviço à população.

\section{AGRADECIMENT0}

Ao CNPq- Bolsista Pos-doc Junior, Apoio-NIPP/UFF (Núcleo Interdisciplinar de Pesquisa de Paisagem) e a Prof. Tânia T. Castellani (UFSC).

\section{REFERÊNCIAS BIBLIOGRÁFICAS}

ALMEIDA, J. R.; SUGUIO, K. Potencialidade geoturística das dunas eólicas da Ilha Comprida - estado de São Paulo. Geociências, v. 31, n. 3, p. 473-484, 2012.

ARAÚJO, M.C.B.; COSTA, M.F. Análise qualiquantitativa do lixo deixado na Baía de Tamandaré-PE-Brasil, por excursionistas. Revista da Gestão Costeira Integrada, v.3, p.58-61, 2003.

BALENSIEFER, M. Recuperação de áreas degradadas no Brasil. In: Conselho Nacional Da Reserva Da Biosfera Da Mata Atlântica - CNRB. Recuperação de áreas degradadas na Mata Atlântica: catálogo bibliográfico. São Paulo. 72 p. 1997.

BERNADI, H.; SEELIGER, U. Population biology of Blutaparon portulacoides (St. Hill.) Mears. on southern Brazilian backshores. Revista Ciência e Cultura, v. 11, n. 41, p.1110-1113, 1989.

BROWER, J. E. E ZAR, J. H. Field and laboratory methods for general ecology. W. C. Brown Company Publishers, Iowa. 1977.

CASTELLANI, T.T. Estrutura e dinâmica populacional de Ipomoea pes-caprae (L.) R. Brown (Convolvulaceae) na Ilha de Santa Catarina. 2003.Tese (Doutorado em Ecologia), Universidade Estadual de Campinas UNICAMP, Campinas, São Paulo.

CORDAZZ0, C.V.; COSTA, C.S.B. Associações vegetais das dunas frontais de Garopaba (SC). Ciência e Cultura, 4l, n.9, p.906-910. 1989.

CORDAZZO, C. V.; SEELIGER, U. Guia ilustrado da vegetação costeira no extremo sul do Brasil. Rio Grande, Ed. FURG, 275 p. 1995.

CORDAZZO, C.V.; SOUZA, H. Z. Germinação de Senecio crassiflorus (compositae). Revista Brasileira de Biologia, v.l, n. 53, p.81-86, 1984.

COSTA, C.S.B.; SEELIGER, U. Quantitative phenology and horizontal distribution of the rhizomatous perennial herb Hydrocotyle bonarienses Lam. in coastal sand dune. Vida Silvestre Neotropical, v.2, n. 2 p. 36-24, 1990.

DE OLIVEIRA, U.R.; DE MELO, A.T.; RIBEIR0, D.; DE SOUZA, D.R.; NEVES, J.; PIETRO-FILHO, J.E.; MUDAT, J.E.; KITHARA, M.V.; HORN-FILHO, N.O. Aspectos granulométricos dos sedimentos siliciclásticos do sistema praia - duna frontal da folha Sombrio, sul do Estado de Santa Catarina, Brasil. Geosul, Florianópolis, v. 22, n. 43, p 123-146, 2007.

FALKENBERG, B. D. Aspectos da flora e da vegetação secundária da restinga de Santa Catarina, sul do Brasil. Insula, v.28, p1-30. 1999. 
FUNBIO - FUNDO BRASILEIR0 PARA A BIODIVERSIDADE; SMAC - SECRETARIA DE MEIO AMBIENTE DA CIDADE DO RIO DE JANEIRO. Plano de Manejo do Parque Natural da Prainha e Parque Natural Municipal de Grumari. Rio de Janeiro, 2012.

G0MES-NET0, A.; EMÍLI0, T.C.; CUNHA, R.S. Plantio de ipomoea pes-caprae nas dunas da praia brava (itajaí, sc): comparação de duas técnicas. Notas Técnicas. Facimar, v.8, p. 33-38, 2004.

HESP, P.A. Review of biological and geomorphological process involved in the initiation and development of incipient foredune. Royal Society of Edimburges. v.96, p.181-200. 1989.

JESUS, R.M. Restauração florestal na mata atlântica. In:Simpósio nacional de recuperação de áreas degradadas, 1997, Ouro Preto, MG. Anais. São Paulo: SOBRADE, UFV. p. 544-558.

LANDIM, P.M.B. Análise estatística de dados geológicos multivariados. Lab. Geomática, DGA, IGCE, UNESP/Rio Claro, Texto didático, v.3, p.128. 2000.

LEITE, A.V.L.; ANDRADE, L.H.C. Riqueza de espécies e composição florística em um ambiente de duna após 50 anos de pressão antrópica: um estudo na Praia de Boa Viagem, Recife, PE - Brasil. Biotemas, v.17, n.1, p. 29 46, 2004.

MADZENA, A.; LASIAK, T. Spatial and temporal variations in beach litter on the Transkei Coast of South Africa. Marine Pollution Bulletin, v. 34, n.11, p. 900-907, 1997.

MARTINS, S.E.; ROSSI, L.; SAMPAIO, P.S.P.; MAGENTA, M.A.G. Caracterização florística de comunidades vegetais de restinga em Bertioga, SP, Brasil. Acta Botanica Brasilica, v.22, n.l, p.249-274. 2008.

MENEZES, L. F. T.; ARAÚJ0, D. S. D. Estrutura de duas formações vegetais do cordão externo da Restinga de Marambaia, RJ. Acta Botanica Brasilica, v.2, n.13, p.223-235. 1999.

MOORE, S.L.; GREGORIO, D.; CARREON, M., WEISBERG, S. B.; LEECASTER, M. K. Composition and distribution of beach debris in Orange County, California. Marine Pollution Bulletin, v.42, n.3, p.241-245, 2001.

MORENO-GASASOLA, P.; EPEJEL, I. Classification and ordination of coastal sand dune vegetation along the gulf and Caribbean sea of Mexico. Vegetation, v.66, p147-182. 1982.

MUEHE, D., BELLIGOTTI, F.M.; LINS-DE-BARROS, F.M.; OLIVEIRA, J.F.; MAIA, L.F.P.G. Potential vulnerability to climate change of the beach-dune system of the Peró coastal plain - Cabo Frio, Rio de Janeiro state, Brazil. Pan-American Journal of Aquatic Sciences, vol. 5, n.2, pp. 267-276. 2010.

MUELLER-DOMBOIS, D.; ELLENBERG, H. Aims and methods of vegetation ecology. New York: J. Wiley. 547 p. 1974.

NORDSTROM, K. F. Beach and dune restoration. São Paulo. Ed. Oficina de textos. 258p. 2008.

PEIXOTO, J. R. V. Interação praia-duna e sua influência sobre a vegetação de duna frontal na costa leste da Ilha de Santa Catarina, SC, Brasil. 2010. Tese (Doutorado em Geografia), Universidade Federal de Santa Catarina, Santa Catarina.

R0MARIZ, D.A. Aspecto da vegetação do Brasil. 2a edição, São Paulo, Brasil. 60p. 1997.

R0SA, L. S.; CORDAZZ0, C. V. Perturbações antrópicas na vegetação das dunas da praia do cassino (RS). Cadernos de Ecologia Aquática, v.2, n.2, p.1-12. 2007.

SANTOS J.N.S. Relação entre morfologia e dieta e uso da macroinfauna por pampos Trachinotus carolinus e Trachinotus goodei (Actinopterygii, Carangidae) em duas praias arenosas do sudeste do Brasil. 2010.Tese (Doutorado em Ciências). Universidade Federal Rural do Rio de Janeiro. Seropédica,.

SCHERER-WIDMER, M. Dune revegetation with native species of restinga on the southern coast of Brazil. Journal of Coastal Research. Special Issue, v.34, p. 593-596. 2001. 
SILVA, T.L.B.; DE AZEVEDO, V.M.; MACHADO, C.B.B.; SOARES, M.A. Parâmetros da biodiversidade nas unidades de conservação da zona oeste do município do Rio de Janeiro - RJ. Novo Enfoque: Caderno de Saúde e Meio Ambiente. Rio de Janeiro, v.21, p.14-19. 2016.

WENTWORTH, C.R. A scale of grade and class terms of clastic sediments. Journal of Geology, v.3, p.377-392. 1922.

ZAMITH, L.R.; DALMASO, V. Revegetação de restingas degradadas no município do Rio de Janeiro, RJ. In: SIMPÓSIO DE ECOSSISTEMAS BRASIlEIROS: CONSERVAÇÃ0, 5, São Paulo, 2000. Anais. São Paulo: Academia de Ciências do Estado de São Paulo, v.4, p.227-234.

ZAMITH R.L.; SCARARANO F.R. Produção de mudas de espécies das restingas do Município do Rio de Janeiro, RJ, Brasil. Acta bot. Bras., v.1, n.18, p.161-176, 2004. 